\title{
BMJ
}

\section{All cause and disease specific mortality in patients with knee or hip osteoarthritis: population based cohort study}

\author{
Eveline Nüesch, research fellow, ${ }^{1,2}$ Paul Dieppe, professor of clinical education research, ${ }^{3}$ Stephan \\ Reichenbach, rheumatologist and senior research fellow, ${ }^{1,4}$ Susan Williams, research associate, ${ }^{5}$ Samuel Iff, \\ research fellow, ${ }^{1,2}$ Peter Jüni, professor of clinical epidemiology ${ }^{1,2}$
} \begin{tabular}{l} 
Institute of Social and Preventive \\
Medicine (ISPM), University of \\
Bern, Switzerland \\
${ }^{2}$ CTU Bern, Bern University \\
Hospital, Bern \\
\hline${ }^{3}$ Institute of Clinical Education, \\
Peninsula Medical School, \\
Universities of Exeter and \\
Plymouth, UK \\
\hline${ }^{4}$ Department of Rheumatology, \\
Clinical Immunology and \\
Allergology, Bern University \\
Hospital
\end{tabular}

${ }^{5}$ Department of Social Medicine, University of Bristol, Bristol, UK Correspondence to: $P$ Jüni juni@ispm.unibe.ch

Cite this as: BMJ 2011;342:d1165 doi:10.1136/bmj.d1165

\section{ABSTRACT}

Objective To examine all cause and disease specific mortality in patients with osteoarthritis of the knee or hip. Design Population based cohort study.

Setting General practices in the southwest of England. Participants 1163 patients aged 35 years or over with symptoms and radiological confirmation of osteoarthritis of the knee or hip.

Main outcome measures Age and sex standardised mortality ratios and multivariable hazard ratios of death after a median of 14 years' follow-up.

Results Patients with osteoarthritis had excess all cause mortality compared with the general population (standardised mortality ratio $1.55,95 \%$ confidence interval 1.41 to 1.70 ). Excess mortality was observed for all disease specific causes of death but was particularly pronounced for cardiovascular (standardised mortality ratio $1.71,1.49$ to 1.98 ) and dementia associated mortality $(1.99,1.22$ to 3.25$)$. Mortality increased with increasing age ( $P$ for trend $<0.001$ ), male sex (adjusted hazard ratio $1.59,1.30$ to 1.96$)$, self reported history of diabetes $(1.95,1.31$ to 2.90$)$, cancer $(2.28,1.50$ to 3.47$)$, cardiovascular disease $(1.38,1.12$ to 1.71$)$, and walking disability $(1.48,1.17$ to 1.86$)$. However, little evidence existed for increased mortality associated with previous joint replacement, obesity, depression, chronic inflammatory disease, eye disease, or presence of pain at baseline. The more severe the walking disability, the higher was the risk of death ( $P$ for trend 10.001 ).

Conclusion Patients with osteoarthritis are at higher risk of death compared with the general population. History of diabetes, cancer, or cardiovascular disease and the presence of walking disability are major risk factors. Management of patients with osteoarthritis and walking disability should focus on effective treatment of cardiovascular risk factors and comorbidities, as well as on increasing physical activity.

\section{INTRODUCTION}

The morbidity associated with chronic rheumatic diseases is well documented, and arthritis is the leading cause of chronic pain and physical disability in the community. ${ }^{1}$ Mortality associated with arthritis is less well characterised. ${ }^{2}$ However, inflammatory disorders, such as rheumatoid arthritis, may result in excess mortality. ${ }^{3}$ Estimates of the standardised mortality ratio in rheumatoid arthritis vary from about 1.3 to 3.0. ${ }^{4}$ The main reason for this excess mortality is cardiovascular disease, although deaths from infections, respiratory diseases, and gastrointestinal disorders are also increased. ${ }^{4}$ The excess in cardiovascular mortality in rheumatoid arthritis is thought to be in part a result of vascular disease being a component of the general inflammatory process and in part a result of drug treatments such as steroids and non-steroidal anti-inflammatory drugs. ${ }^{5}$ Immobility resulting from arthritis may also shorten lifespan; for example, a recent study showed that disability in older people was an important risk factor for increased mortality independent of age and comorbid conditions. ${ }^{6}$

Very little is known about mortality in osteoarthritis, although it is by far the most common form of rheumatic disease. ${ }^{7}$ Osteoarthritis is not an inflammatory condition and is treated with anti-inflammatory drugs rather than with steroids. ${ }^{8}$ The condition often results in immobility, ${ }^{9}$ and obesity is a major comorbidity in patients with osteoarthritis. ${ }^{10}$ Thus, reasons exist for thinking that people with osteoarthritis may have an excess in mortality as well as in morbidity. Hochberg recently reviewed the literature on mortality in osteoarthritis. ${ }^{2}$ He found seven studies, several of which suggested an increased risk of death in people with osteoarthritis, ${ }^{11-18}$ but these studies had several methodological problems. ${ }^{2}$

Osteoarthritis is a common cause of medical consultation. ${ }^{19} \mathrm{~A}$ better understanding of the burden of osteoarthritis in terms not only of symptoms but also of a reduced life expectancy, and an investigation into potentially modifiable risk factors, could inform future management strategies beyond the current focus on treating the symptoms. ${ }^{8}$ Therefore, we used data from a large population based study to determine whether people with osteoarthritis of the knee or hip are subject to an increased risk of death and to examine the causes and potential risk factors. ${ }^{2021}$

\section{METHODS}

\section{Patients}

The Somerset and Avon Survey of Health is a population based study of 28080 people aged 35 years or over 
randomly selected from 40 general practices in the south west of England.$^{2021}$ The survey excluded 2034 people who had moved out of the study area, had a severe mental or terminal illness, or had died. The remaining 26046 people were sent a screening questionnaire comprising questions on general health, use of health services, and symptoms of hip and knee disease. Non-respondents were sent two reminders and contacted by phone, if necessary. ${ }^{22} 23$ People were screened for knee and hip pain with a modified version of the question used in the first National Health and Nutrition Examination Survey. ${ }^{10}$ The invitation for clinical examination and examination of patients with knee or hip pain was organised in two phases according to the geographical location of the participating practices in 1994-5.

\section{Procedures}

We used data collected at baseline on demographic and socioeconomic characteristics, self reported health

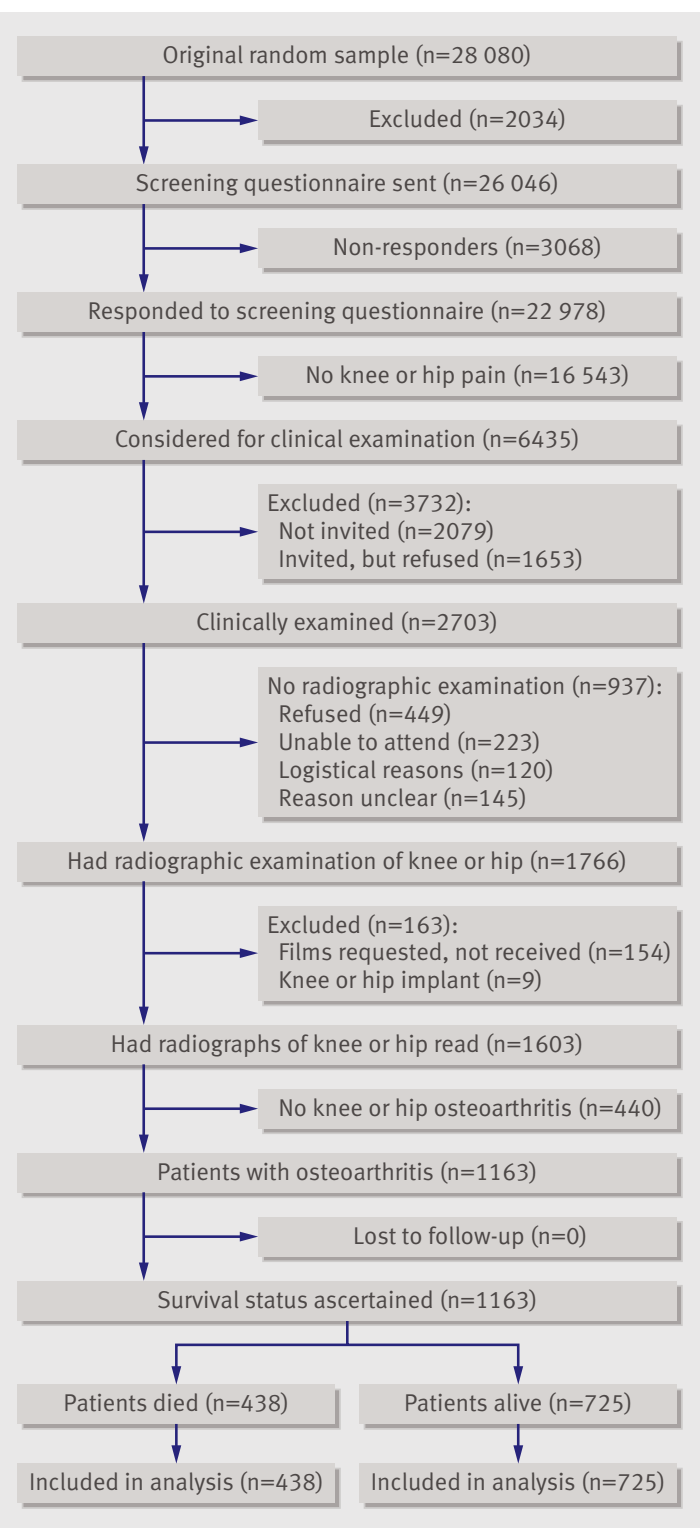

Fig 1| Flow of participants through different stages of study conditions and drug use, and presence of knee or hip pain and walking disability as potential predictors of mortality. Participants reported presence or absence of several comorbidities, which were grouped as follows: cardiovascular disease (angina, myocardial infarction, heart failure, claudication, and stroke), cancer, chronic obstructive pulmonary disease (including asthma, bronchitis, and emphysema), arterial hypertension, diabetes, chronic inflammatory disease (including rheumatoid arthritis), eye disease (cataract or glaucoma), and depression. We classified body mass index $\left(\mathrm{kg} / \mathrm{m}^{2}\right)$ into two categories: non-obese $(<30)$ and obese $(\geq 30)$. We classified the self reported use of oral analgesics into use of paracetamol, non-steroidal anti-inflammatory drugs, and opioids, irrespective of the dosage.

In 1994-5, examined participants were asked about knee or hip pain with the following questions: "In the past 12 months, have you had pain in or around your left (right) knee (hip) on most days for one month or longer during the day (night)? (yes/no)." We considered participants to have pain if they reported pain during the day or night in at least one hip or knee. ${ }^{24}$ Participants were asked about their walking disability: "How difficult is it for you to go outdoors and walk down the road on your own? (not difficult/quite difficult/very difficult/impossible)." ${ }^{24}$ We considered patients to have disability if walking was described at least to be "quite difficult." Survival status of the 2703 participants and information on the cause of death up to 9 February 2009 came from the Office for National Statistics, London. The causes of death were registered and coded according to ICD-9 (international classification of diseases, 9th revision) or ICD-10. We recoded all to ICD-10 and considered death to be caused by the primary cause of death listed on the death certificate with ICD-10 codes of "I" for cardiovascular disease, "C" for cancer, "J" for respiratory disease, "K" for gastrointestinal disease, and "F01," "F03," or "R54" for dementia associated disease.

At baseline, participants had weight bearing anteroposterior and lateral radiographs of the knees and hip according to a standardised protocol. Details of the radiographic examinations are described elsewhere. ${ }^{24}$ We assigned Kellgren-Lawrence grades of global radiological severity on anteroposterior views by using a scale from 0 to $4(0=$ no features of osteoarthritis; $1=$ minute osteophytes of doubtful significance; $2=$ definite osteophytes, no definite joint space narrowing; $3=$ definite joint space narrowing of a moderate degree; 4=severe joint space impairment). ${ }^{25} \mathrm{We}$ considered osteoarthritis to be present if a KellgrenLawrence grade of at least 1 was measured for at least one joint.

\section{Statistical analysis}

We compared mortality between included patients with osteoarthritis of the knee or hip and the general population in England and Wales by calculating standardised mortality ratios (the ratio of observed to expected number of deaths) and corresponding 95\% 
Table 1|Age and sex standardised mortality ratios

\begin{tabular}{|c|c|c|c|c|c|c|c|c|c|}
\hline \multirow[b]{3}{*}{ Cause of death } & \multicolumn{3}{|c|}{ All patients $(n=1163)$} & \multicolumn{3}{|c|}{ Men $(n=503)$} & \multicolumn{3}{|c|}{ Women $(n=660)$} \\
\hline & \multicolumn{2}{|c|}{ No of deaths } & \multirow[b]{2}{*}{ SMR $(95 \% \mathrm{Cl})$} & \multicolumn{2}{|c|}{ No of deaths } & \multirow[b]{2}{*}{ SMR $(95 \% \mathrm{Cl})$} & \multicolumn{2}{|c|}{ No of deaths } & \multirow[b]{2}{*}{ SMR $(95 \% \mathrm{Cl})$} \\
\hline & Observed & Expected & & Observed & Expected & & Observed & Expected & \\
\hline All causes & 438 & 283 & 1.55 (1.41 to 1.70$)$ & 204 & 129 & 1.58 (1.38 to 1.81$)$ & 234 & 154 & 1.52 (1.34 to 1.73$)$ \\
\hline Cardiovascular disease & 188 & 110 & 1.71 (1.49 to 1.98$)$ & 95 & 52 & 1.82 (1.49 to 2.22$)$ & 93 & 57 & $1.62(1.32$ to 1.98$)$ \\
\hline Cancer related & 123 & 93 & 1.32 (1.10 to 1.57$)$ & 58 & 44 & 1.33 (1.03 to 1.72$)$ & 65 & 50 & 1.31 (1.03 to 1.67$)$ \\
\hline Respiratory disease & 43 & 33 & 1.29 (0.96 to 1.74$)$ & 20 & 15 & $1.38(0.89$ to 2.14$)$ & 23 & 19 & $1.22(0.81$ to 1.84$)$ \\
\hline Gastrointestinal disease & 19 & 13 & 1.47 (0.94 to 2.30$)$ & 6 & 6 & 1.09 (0.49 to 2.42$)$ & 13 & 7 & 1.75 (1.02 to 3.01$)$ \\
\hline Dementia associated & 16 & 8 & 1.99 (1.22 to 3.25$)$ & 5 & 2 & $2.29(0.95$ to 5.50$)$ & 11 & 6 & 1.88 (1.04 to 3.39$)$ \\
\hline
\end{tabular}

SMR=standardised mortality ratio.

confidence intervals. Expected numbers of deaths came from age and sex specific mortality data provided in the mortality statistics from 2002 (Office for National Statistics, London) in 10 year age bands, and we used the age at the mid-point of the observation time to assign study patients to age bands. In univariable and multivariable Cox proportional hazards models, we examined associations between all cause and disease specific mortality and characteristics of patients at baseline, and we tested associations with two sided Wald tests. We accounted for missing data in the

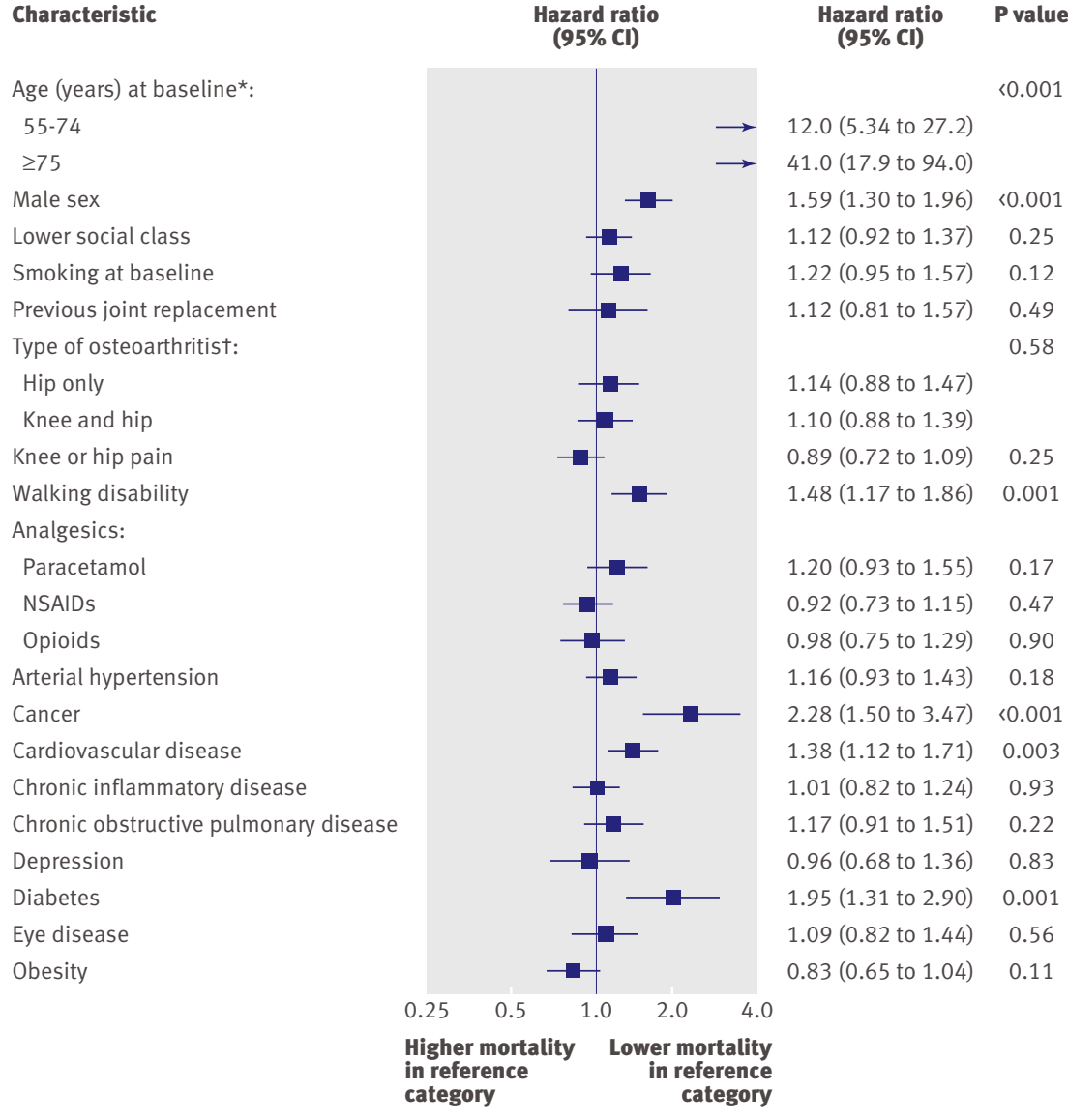

Fig 2| Associations between characteristics at baseline and all cause mortality up to 15 years thereafter. Shows hazard ratios with corresponding $95 \%$ confidence intervals from multivariable Cox proportional hazards models after multiple imputation of missing values in covariates. $P$ values were calculated by using two sided Wald tests. NSAIDs=non-steroidal antiinflammatory drugs. *Age 35-54 as reference category. †lsolated knee osteoarthritis as reference category baseline covariates by using multiple imputation with overall mortality, log transformed survival time, sex, age, social class, smoking, comorbid conditions, use of analgesics, previous joint replacement, type of osteoarthritis, knee or hip pain and walking disability, and study characteristics such as recruitment cycle as variables in the imputation model, to create 20 imputed datasets. ${ }^{26-28}$

In sensitivity analyses, we examined associations between overall mortality and baseline characteristics by using multivariable Cox proportional hazards regression models restricted to patients with radiographic osteoarthritis defined by a more stringent Kellgren-Lawrence grade of at least 2 and restricted to patients with complete information on covariates. Nelson-Aalen estimates of cumulative hazards and statistical tests based on Schoenfeld residuals indicated that proportional hazards assumptions were satisfied, and Cox-Snell residuals indicated a satisfactory model fit. Finally, we compared numbers and percentages of characteristics at baseline between participants who were invited but did not attend, those who attended clinical examination only, and those with clinical and radiographic examination. All $\mathrm{P}$ values and 95\% confidence intervals are two sided.

\section{RESULTS}

Figure 1 shows the flow of participants through the study. Of 22978 responders to the screening questionnaire, 6435 participants were considered, 4356 were invited for a clinical examination, and 2703 attended; $1766(65 \%)$ had a radiographic examination of their knees or hips, and we included 1163 (43\%) patients with radiologically confirmed osteoarthritis of the knee or hip in the analyses. Web appendix 1 shows a comparison of participants who were invited but did not attend, those who attended for a clinical examination only, and those with clinical and radiographic examination. Participants who had radiographic examination were younger than the remainder of participants. No relevant differences existed for the other characteristics at baseline.

At the end of follow-up, $725(62 \%)$ of the included patients were still alive; $188(16 \%)$ died from cardiovascular disease, $123(11 \%)$ died from cancer, 43 $(3.7 \%)$ died from respiratory disease, 19 (1.6\%) died from gastrointestinal disease, $16(1.4 \%)$ died from 
Table 2 |Characteristics of patients at baseline. Values are numbers (percentages) unless stated otherwise

\begin{tabular}{|c|c|c|c|c|}
\hline \multirow[b]{2}{*}{ Characteristic at baseline } & \multicolumn{2}{|c|}{ Patients died } & \multirow[b]{2}{*}{$\begin{array}{l}\text { Crude hazard ratio* } \\
\qquad(95 \% \mathrm{Cl})\end{array}$} & \multirow[b]{2}{*}{ Pvalue* } \\
\hline & $\begin{array}{c}\text { Yes } \\
(n=438)\end{array}$ & $\begin{array}{c}\text { No } \\
(n=725)\end{array}$ & & \\
\hline \multicolumn{4}{|l|}{ Age (years) at baseline: } & \multirow{4}{*}{$<0.001$} \\
\hline $35-54$ & $6(1)$ & $169(23)$ & 1.00 (reference) & \\
\hline $55-74$ & $273(62)$ & $503(69)$ & $12.4(5.53$ to 27.9$)$ & \\
\hline$\geq 75$ & $159(36)$ & $53(7)$ & $40.7(18.0$ to 92.0$)$ & \\
\hline Male sex & $204(47)$ & $299(41)$ & $1.21(1.00$ to 1.46$)$ & 0.048 \\
\hline Lower social class (IIIM to V) & $228(52)$ & $342(47)$ & $1.21(1.00$ to 1.46$)$ & 0.050 \\
\hline Smoking & $70(16)$ & $115(16)$ & 0.94 (0.74 to 1.19$)$ & 0.60 \\
\hline Previous joint replacement & $42(10)$ & $38(5)$ & 1.61 (1.17 to 2.22$)$ & 0.003 \\
\hline \multicolumn{4}{|l|}{ Type of osteoarthritis: } & \multirow{4}{*}{0.14} \\
\hline Knee only & $130(30)$ & $233(32)$ & 1.00 (reference) & \\
\hline Hip only & $120(27)$ & $222(31)$ & $0.98(0.77$ to 1.26$)$ & \\
\hline Knee and hip & $188(43)$ & $270(37)$ & $1.20(0.96$ to 1.50$)$ & \\
\hline Knee or hip pain & $289(66)$ & $477(66)$ & $1.00(0.82$ to 1.22$)$ & 0.97 \\
\hline Walking disability & $152(35)$ & $136(19)$ & $1.93(1.59$ to 2.36$)$ & $<0.001$ \\
\hline \multicolumn{5}{|l|}{ Type of analgesic: } \\
\hline Paracetamol & $209(48)$ & $269(37)$ & $1.28(1.05$ to 1.57$)$ & 0.015 \\
\hline Non-steroidal anti-inflammatory drugs & $123(28)$ & $216(30)$ & $0.83(0.67$ to 1.03$)$ & 0.09 \\
\hline Opioids & $142(32)$ & $173(24)$ & $1.32(1.07$ to 1.63$)$ & 0.008 \\
\hline Arterial hypertension & $138(32)$ & $166(23)$ & $1.43(1.17$ to 1.75$)$ & 0.001 \\
\hline Cancer & $25(6)$ & $16(2)$ & 2.11 (1.41 to 3.16$)$ & $<0.001$ \\
\hline Cardiovascular disease & $152(35)$ & $131(18)$ & $1.96(1.61$ to 2.38$)$ & $<0.001$ \\
\hline Chronic inflammatory disease & $163(37)$ & $216(30)$ & $1.30(1.07$ to 1.58$)$ & 0.008 \\
\hline Chronic obstructive pulmonary disease & 77 (18) & $110(15)$ & 1.14 (0.89 to 1.46$)$ & 0.29 \\
\hline Depression & $40(9)$ & $58(8)$ & 1.09 (0.78 to 1.50$)$ & 0.62 \\
\hline Diabetes & $29(7)$ & $18(2)$ & $2.10(1.44$ to 3.06$)$ & $<0.001$ \\
\hline Eye disease & $67(15)$ & $39(5)$ & 2.10 (1.62 to 2.73$)$ & $<0.001$ \\
\hline Obesity & $105(24)$ & $220(30)$ & $0.77(0.62$ to 0.96$)$ & 0.020 \\
\hline
\end{tabular}

*Univariable hazard ratios, $95 \%$ confidence intervals, and $\mathrm{P}$ values were derived from Cox regression models after multiple imputation of missing covariate data; hazard ratios $>1$ indicate lower mortality in reference category.

dementia, and $49(4.2 \%)$ died from other diseases. The median length of follow-up among surviving patients was 14.3 (range 13.6-15.2) years.

Table 1 shows the number of observed and expected deaths and the corresponding age and sex standardised mortality ratios for different causes of death. We found excess all cause mortality in patients with radiologically confirmed osteoarthritis of the knee or hip compared with the general population (standardised mortality ratio $1.55,95 \%$ confidence interval 1.41 to 1.70). We found higher rates of death in patients with osteoarthritis compared with the general population for all the different causes of death, but the effect was particularly pronounced for cardiovascular (standardised mortality ratio $1.71,1.49$ to 1.98 ) and dementia associated mortality $(1.99,1.22$ to 3.25$)$. We found little evidence to suggest that age standardised mortality ratios differed between men and women.

Table 2 shows the baseline characteristics of patients according to their survival status at the end of followup. In univariable analyses, we found increased mortality in men; in older patients and those with lower socioeconomic status; in patients with previous joint replacement; in patients with self reported comorbidities such as cardiovascular disease, cancer, arterial hypertension, diabetes, chronic inflammatory disease, and eye disease; and in patients who reported the use of paracetamol or opioids $(\mathrm{P} \leq 0.05)$. Patients with knee or hip pain were not more likely to die than were patients without pain $(\mathrm{P}=0.97)$, but patients with walking disability were more likely to die than were those without (hazard ratio 1.93, 95\% confidence interval 1.59 to $2.36 ; \mathrm{P}<0.001)$.

Figure 2 shows results from multivariable analysis on the association between baseline characteristics and all cause mortality. The risk of death seemed to be unaffected by type of osteoarthritis $(\mathrm{P}=0.58)$, previous joint replacement $(\mathrm{P}=0.49)$, obesity $(\mathrm{P}=0.11)$, intake of different types of conventional analgesics or non-steroidal anti-inflammatory drugs at baseline $(\mathrm{P} \geq 0.17)$; self reported history of depression $(\mathrm{P}=0.83)$, chronic inflammatory disease $(\mathrm{P}=0.93)$, or eye disease $(\mathrm{P}=0.56)$; and presence of hip or knee pain at baseline $(\mathrm{P}=0.25)$. Conversely, a self reported history of diabetes (hazard ratio $1.95,1.31$ to 2.90$)$, cancer $(2.28$, 1.50 to 3.47$)$, or cardiovascular disease $(1.38,1.12$ to 1.71) and the presence of walking disability at baseline $(1.48,1.17$ to 1.86$)$ were independently associated with increased all cause mortality.

Figure 3 (top left) shows the cumulative incidence of death from all causes in patients with and without walking disability at baseline: $53 \%$ of patients with walking disability compared with $33 \%$ of patients without disability died. As indicated in table 3 , the crude hazard ratio of 1.93 ( 1.59 to $2.36 ; \mathrm{P}<0.001$ ) was attenuated to 1.58 (1.29 to 1.93) after adjustment for age and sex and to 1.48 after full adjustment as reported above. In a sensitivity analysis, the 88 patients with severe walking disability had a higher risk of death from any cause (fully adjusted hazard ratio $1.88,1.37$ to 2.56 ) than did the 200 patients with moderate walking disability $(1.31,1.00$ to 1.70 ; $\mathrm{P}$ for trend $<0.001)$. Figure 3 shows that deaths from cardiovascular causes were higher in patients with walking disability $(25 \%$ v $12 \%$; crude hazard ratio $2.40,1.79$ to $3.23 ; \mathrm{P}<0.001)$. Table 3 indicates that the association between deaths from cardiovascular causes and walking disability remained after adjustment for baseline covariates, although it was attenuated (fully adjusted hazard ratio $1.72,1.22$ to 2.41 $\mathrm{P}=0.002)$. Patients with walking disability also had higher rates of deaths from respiratory causes $(4.9 \% \mathrm{r}$ $3.4 \%$, fully adjusted hazard ratio $1.74,0.82$ to 3.71 ; $\mathrm{P}=0.15)$ and gastrointestinal causes $(3.5 \%$ v $1.3 \%$; 2.51, 0.89 to $7.14 ; \mathrm{P}=0.08)$ compared with patients without disability, but confidence intervals were wide and $\mathrm{P}$ values non-significant. Rates for cancer related death $(11 \%$ v 11\%; $\mathrm{P}=0.93)$ and death associated with dementia $(1.4 \%$ v $1.6 \% ; \mathrm{P}=0.58)$ were similar between patients with and without walking disability at baseline examination.

The web appendix shows results from sensitivity analyses of all cause mortality. When we restricted the analysis to the $885(76 \%)$ patients with radiographic osteoarthritis of a Kellgren-Lawrence grade of 2 or above, we found the associations between baseline 
$\overline{\text { Table } 3 \mid \text { Associations between walking disability and mortality }}$

\begin{tabular}{|c|c|c|}
\hline & $\begin{array}{c}\text { Hazard ratio } \\
(95 \% \mathrm{Cl})^{\star}\end{array}$ & Pvalue* \\
\hline \multicolumn{3}{|l|}{ All cause mortality: } \\
\hline Crude & $1.93(1.59$ to 2.36$)$ & $<0.001$ \\
\hline Age and sex adjusted & $1.58(1.29$ to 1.93$)$ & $<0.001$ \\
\hline Fully adjusted & $1.48(1.17$ to 1.86$)$ & 0.001 \\
\hline \multicolumn{3}{|c|}{ Death from cardiovascular causes: } \\
\hline Crude & 2.40 (1.79 to 3.23$)$ & $<0.001$ \\
\hline Age and sex adjusted & 1.95 (1.44 to 2.63$)$ & $<0.001$ \\
\hline Fully adjusted & $1.72(1.22$ to 2.41$)$ & 0.002 \\
\hline \multicolumn{3}{|l|}{ Cancer related death: } \\
\hline Crude & $1.22(0.82$ to 1.82$)$ & 0.34 \\
\hline Age and sex adjusted & 1.06 (0.70 to 1.58$)$ & 0.80 \\
\hline Fully adjusted & 0.98 (0.62 to 1.56$)$ & 0.93 \\
\hline \multicolumn{3}{|c|}{ Death from respiratory causes: } \\
\hline Crude & $1.74(0.90$ to 3.34$)$ & 0.10 \\
\hline Age and sex adjusted & 1.37 (0.70 to 2.66$)$ & 0.36 \\
\hline Fully adjusted & $1.74(0.82$ to 3.71$)$ & 0.15 \\
\hline \multicolumn{3}{|c|}{ Death from gastrointestinal causes: } \\
\hline Crude & $3.26(1.32$ to 8.06$)$ & 0.010 \\
\hline Age and sex adjusted & $2.46(0.98$ to 6.20$)$ & 0.06 \\
\hline Fully adjusted & $2.51(0.89$ to 7.14$)$ & 0.08 \\
\hline \multicolumn{3}{|c|}{ Death associated with dementia: } \\
\hline Crude & 1.15 (0.37 to 3.63$)$ & 0.81 \\
\hline Age and sex adjusted & 0.68 (0.21 to 2.19$)$ & 0.52 \\
\hline Fully adjusted $\dagger$ & 0.69 (0.18 to 2.59$)$ & 0.58 \\
\hline \multicolumn{3}{|c|}{$\begin{array}{l}\text { *Hazard ratios, } 95 \% \text { confidence intervals, and P values were derived } \\
\text { from Cox proportional hazards models after multiple imputation of } \\
\text { missing covariate data; hazard ratios }>1 \text { indicate higher mortality in } \\
\text { patients with walking disability at baseline. } \\
\text { †Not adjusted for history of obesity, diabetes, depression, and cancer } \\
\text { owing to collinearity. }\end{array}$} \\
\hline
\end{tabular}

characteristics and all cause mortality to be robust (web appendix 2). Similarly, results were mostly unaffected by a restriction of the analysis to participants with complete information (web appendix 3).

\section{DISCUSSION}

In this large population based cohort study, we found that people with symptoms and radiographical confirmation of osteoarthritis of the hip or knee have an excess all cause mortality compared with the general population. The excess was particularly pronounced for death from cardiovascular causes and for dementia associated mortality. Predictors of excess mortality were diabetes, cardiovascular disease, and cancer reported at baseline. Conversely, we did not find robust associations with the reported use of analgesics and nonsteroidal anti-inflammatory drugs, obesity, or joint replacement at baseline. The most striking finding, however, was the strong relation between excess mortality and walking disability. Our analysis suggested that the more severe the restriction in walking ability the more likely a person was to die early. Most of the excess mortality associated with walking problems was due to cardiovascular causes. We also saw a relation with deaths from gastrointestinal disorders, but the numbers were small and some of this may be explained by confounders such as total use of non-steroidal antiinflammatory drugs or alcohol consumption.

\section{Strengths and weaknesses}

The strengths of this study include the population based design of the cohort, ${ }^{2021}$ the large number of patients with osteoarthritis included, and the long term followup of patients for a median of 14 years. We were able to ascertain the survival status of all patients included in our study. However, we cannot rule out residual selection bias introduced by participants refusing clinical or radiographic examination. In our analyses, we used baseline characteristics - namely, comorbidities and analgesic/anti-inflammatory consumption - that were reported by patients and information given on death certificates to derive estimates for disease specific mortality. This might have resulted in misclassifications. If misclassifications were non-differential, the observed associations are likely to be underestimates. We cannot exclude, however, that misclassifications were differential, which could bias estimates in either direction. The most important limitation of this study is the missing data for baseline covariates, which we accounted for by using multiple imputation. This procedure is based on the assumption that no unobserved characteristics exist that affect missingness. ${ }^{27}$ Results are valid only if this assumption is not violated, which is difficult to verify. As an alternative to multiple imputation, we did a sensitivity analysis restricted to patients with complete data for covariates and found similar results. A final limitation is that we were able to analyse drug use only with data collected at baseline, which are unlikely to be representative of the cumulative use of drugs over the entire study period. This is likely to explain the observed lack of evidence for an association between use of non-steroidal anti-inflammatory drugs and mortality. Finally, any radiological definition of osteoarthritis may be considered arbitrary and debatable. We chose to base our definition primarily on the presence of grade 1 osteophytes as required for a Kellgren-Lawrence grade of 1 and as used previously in this study and others. ${ }^{2429}$ When we used an alternate definition of osteoarthritis, however, we found much the same results (web appendix 2).

\section{Context}

Although the primary focus in research on osteoarthritis has been on pain and disability, some previous studies have provided evidence that mortality is increased in people with osteoarthritis. ${ }^{2}$ Previous studies had several important limitations. Three studies recruited only from hospitals or medical practices, ${ }^{111214}$ one study included only women with a specific employment history, ${ }^{13}$ and another investigated patients after total knee replacement. ${ }^{17}$ Of the two population based studies, one reported prevalence and the other reported incidence but had a small sample size. ${ }^{12}{ }^{1416}$ Only three studies reported major causes of death, ${ }^{11} 1317$ and only two studies described factors associated with an increased risk of death. ${ }^{1316}$ Four studies did not adjust analyses for important prognostic factors such as 

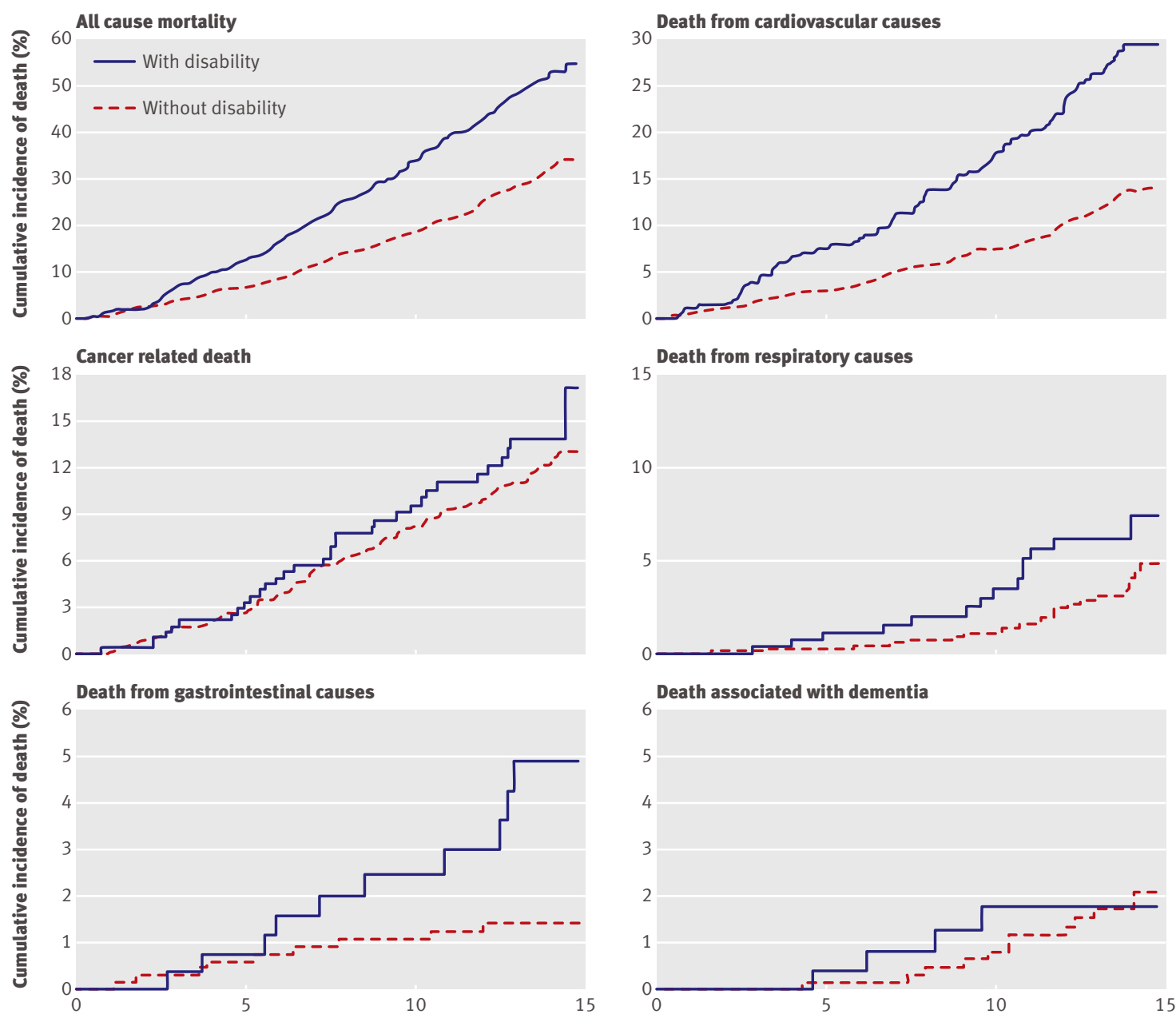

No at risk

Years after baseline examination

Years after baseline examination

Without disability

711

With disability

288

663

578

111

711

663

578

111

Fig 3 | All cause and disease specific mortality in patients with and without walking disability at baseline examination. KaplanMeier curves show the cumulative incidence of all cause mortality, death from cardiovascular causes, cancer related death, death from respiratory causes, death from gastrointestinal causes, and death associated with dementia up to 15 years

smoking history or obesity. ${ }^{11} 121518$ Ours is the first study to investigate patterns and causes of incident mortality in a large population based sample of people with osteoarthritis, overcoming many of the limitations of previous studies.

\section{Explanations}

Most deaths were due to cardiovascular causes, and the association between deaths from all causes and walking disability is driven largely by deaths due to cardiovascular causes. We found no evidence that cancer related deaths and deaths associated with dementia are related to increased walking disability, which suggests that other pathways are more relevant in these cases. Two main possible explanations exist for our findings. Firstly, reduced physical activity may lead to reduced protection against cardiovascular disease. Alternatively, patients with osteoarthritis may have chronic smouldering inflammation with increased levels of inflammatory cytokines, such as increased circulating concentrations of interleukin 6 and tumour necrosis factor $\alpha$, possibly as a result of ongoing tissue damage. ${ }^{3031}$ This chronic inflammation may be causally involved in various chronic conditions, such as cardiovascular and neurodegenerative disease, diabetes, or cancer. ${ }^{32}$ We found increased rates of cardiovascular, cancer, and dementia associated deaths, which would be fully concordant with this reasoning. Wagner et al recently reported an increased risk of cancer in patients with arthroplasty compared with the general population. ${ }^{33}$ Although the authors suggested that this increase in risk may be caused by the prosthesis material, we hypothesise that arthroplasty was a mere surrogate marker for osteoarthritis, which in turn was associated with chronic inflammation and subsequent increase in the risk of cancer. A third possibility is that the use of non-steroidal anti-inflammatory drugs is a major factor in the excess mortality of 


\section{WHAT IS ALREADY KNOWN ON THIS TOPIC}

Osteoarthritis is the most common rheumatic disease in elderly people

Morbidity associated with osteoarthritis has been extensively studied, but associated mortality is less well documented

Several studies have suggested an increased risk of death in people with osteoarthritis, but many of these studies had methodological problems

\section{WHAT THIS STUDY ADDS}

Patients with osteoarthritis have an excess mortality compared with the general population

Major risk factors associated with increased mortality in osteoarthritis patients are walking disability and a history of cancer, diabetes, and cardiovascular disease

Patients with osteoarthritis and walking disability are at increased risk of death from cardiovascular causes, so management of such patients should focus on effective treatment of cardiovascular risk factors

people with osteoarthritis. Although our findings do not directly support this, a recently published network meta-analysis by our group indicates that this may be the case. ${ }^{34}$ Six out of seven non-steroidal anti-inflammatory drugs were associated with clinically relevant twofold to fourfold increases in the risk of myocardial infarction, stroke, or cardiovascular death compared with placebo. ${ }^{34}$

We found a protective effect of obesity on overall mortality in univariable analysis and-albeit statistically non-significant-in multivariable analysis. This phenomenon is known as the obesity paradox: whereas obesity itself is associated with an increased risk of developing conditions such as coronary artery disease or hypertension, once the condition is manifest, obesity seems to be protective..$^{35}$ Explanations include lower vascular resistance and plasma renin activity in obese patients with an established condition that is in turn associated with cardiovascular events, compared with lean patients with the same condition. ${ }^{35}$ Alternatively, obese and non-obese patients may have different disease phenotypes resulting in better prognosis in obese patients: cardiovascular conditions that are mainly caused by obesity and associated risk factors may have a more favourable prognosis than cardiovascular conditions in non-obese patients with a strong genetic predisposition for the condition..$^{35}$ The paradox was established for a range of conditions and phenotypes, including old age and rheumatoid arthritis. To our knowledge, however, we are the first to establish the obesity paradox for mortality in patients with osteoarthritis.

\section{Implications}

We believe that these data have important implications for the ways in which people presenting with osteoarthritis should be managed. If a patient has symptoms of osteoarthritis with associated walking disability, then he or she is at a clearly increased risk of premature death from cardiovascular disease. This means that healthcare professionals seeing such people should assess and consider treating all cardiovascular risk factors, including hypertension, hyperlipidaemia, smoking, and physical inactivity. We suggest that doctors should approach the management of their patients with osteoarthritis much as they would for those who present with gout - that is, by using the presenting condition as a red flag for premature cardiovascular death. ${ }^{36-38}$ Wagenmakers et al put forward such an approach in the context of joint replacement. ${ }^{39}$ These authors suggested that the aim of an intervention for osteoarthritis such as joint replacement should be to get people back to a level of activity that would fit with the World Health Organization's recommendations for minimum exercise required to help prevent cardiovascular disease and developed a measurement instrument to assess such an outcome. ${ }^{39}$ Increased physical activity has been suggested to reduce the overall mortality and risk of ischaemic heart disease in men, ${ }^{4041}$ and it is known to be of value to people with osteoarthritis in many other ways, reducing pain and depression, for example. ${ }^{42-45}$ An aggressive approach to helping people to become more active, in the face of painful osteoarthritis, thus seems fully justified.

\section{Conclusions}

In this large population based study, we found an excess mortality in people with symptoms and radiographic confirmation of osteoarthritis of the knee or hip compared with the general population, irrespective of the cause of death. The most important risk factors for all cause mortality in osteoarthritis (besides older age and male sex) were a history of diabetes, cardiovascular disease, or cancer and increased walking disability. In patients with walking disability, the risk of death from cardiovascular causes was increased. We conclude that the management of patients with osteoarthritis and walking disability should focus on effective treatment of cardiovascular risk factors and comorbidities and on helping people to increase their level of physical activity.

We thank all study participants and the partners and staff of participating general practices for their support and interest in the study. We are indebted to the whole of the Somerset and Avon Survey of Health Research Team: Kirsty Alchin, Ros Berkeley-Hill, Jane Brooks, Hilary Brownett, Phil Chan, Clare Cross, Catherine Dawe, Cathy Doel, Jenny Eachus, Helen Forward, Matthew Grainge, Fiona Hollyman, Sue Jones, Helen Moore, Kate Morris, Nicky Pearson, Brian Quilty, Chris Smith, Lynne Smith, Gwyn Williams, Mark Williams, and Andrea Wilson; and to Allan Douglas and Doreen Cook at Dillon Computing. Finally, we thank our coinvestigators, Jenny Donovan, Tim Peters, and Stephen Frankel. The Department of Social Medicine is the lead centre for the MRC Health Services Research Collaboration. We are grateful to Pete Shiarly for the management and maintenance of the database.

Contributors: PJ and PD had the idea for the study and were primarily responsible for developing the protocol. PD, SR, SW, and PJ contributed to data collection. EN did the data preparation and analysis. All authors reviewed the protocol and participated in interpretation of data. EN, PD, and PJ wrote the first draft of the paper, and all authors contributed to the final draft. PJ and PD are the guarantors.

Funding: The Somerset and Avon Survey of Health was originally funded by the Department of Health and the South and West NHS Research and Development Directorate. This work was funded by the Swiss National Science Foundation (grant numbers 3233-066377 and 3200-066378) and by Arthritis Research UK. The funding bodies had no role in the design or conduct of the study; collection, management, analysis, or interpretation of the data; or preparation, review, or approval of the manuscript. 
Competing interests: All authors have completed the Unified Competing Interest form at www.icmje.org/coi_disclosure.pdf (available on request from the corresponding author) and declare: no support from any organisation for the submitted work; no financial relationships with any organisations that might have an interest in the submitted work in the previous three years; no other relationships or activities that could appear to have influenced the submitted work.

Ethical approval: The study was reviewed and approved by the relevant local research ethics committees, and all participants provided written informed consent.

Data sharing: No additional data are available.

1 Felson DT, Zhang Y. An update on the epidemiology of knee and hip osteoarthritis with a view to prevention. Arthritis Rheum 1998;41:1343-55.

2 Hochberg MC. Mortality in osteoarthritis. Clin Exp Rheumatol 2008;26:S120-4.

3 Myasoedova E, Davis JM, Crowson CS, Gabriel SE. Epidemiology of rheumatoid arthritis: rheumatoid arthritis and mortality. Curr Rheumatol Rep 2010;12:379-85.

4 Gabriel SE, Michaud K. Epidemiological studies in incidence, prevalence, mortality, and comorbidity of the rheumatic diseases. Arthritis Res Ther 2009;11:229.

5 Snow MH, Mikuls TR. Rheumatoid arthritis and cardiovascular disease: the role of systemic inflammation and evolving strategies of prevention. Curr Opin Rheumatol 2005;17:234-41.

6 Landi F, Liperoti R, Russo A, Capoluongo E, Barillaro C, Pahor M, et al. Disability, more than multimorbidity, was predictive of mortality among older persons aged 80 years and older. J Clin Epidemiol 2010;63:752-9.

7 Murray C, Lopez A, eds. The global burden of disease: a comprehensive assessment of mortality and disability from diseases, injuries, and risk factors in 1990 and projected to 2002. World Health Organization, 1996.

8 Juni P, Reichenbach S, Dieppe P. Osteoarthritis: rational approach to treating the individual. Best Pract Res Clin Rheumatol 2006;20:721-40.

9 McAlindon TE, Cooper C, Kirwan JR, Dieppe PA. Knee pain and disability in the community. Br / Rheumatol 1992;31:189-92.

10 Anderson JJ, Felson DT. Factors associated with osteoarthritis of the knee in the first national Health and Nutrition Examination Survey (HANES I): evidence for an association with overweight, race, and physical demands of work. Am J Epidemiol 1988;128:179-89.

11 Monson RR, Hall AP. Mortality among arthritics. / Chronic Dis 1976;29:459-67.

12 Lawrence RC, Everett DF, Hochberg MC. Arthritis. In: Coroni-Huntley IC, Huntley RR, Feldmann JI, eds. Health status and well-being of the elderly. Oxford University Press, 1990:136-51.

13 Cerhan JR, Wallace RB, el-Khoury GY, Moore TE, Long CR. Decreased survival with increasing prevalence of full-body, radiographically defined osteoarthritis in women. Am J Epidemiol 1995;141:225-34.

14 Haara MM, Manninen P, Kröger H, Arokoski JPA, Kärkkäinen A, Knekt $P$, et al. Osteoarthritis of finger joints in Finns aged 30 or over: prevalence, determinants, and association with mortality. Ann Rheum Dis 2003;62:151-8.

15 Watson DJ, Rhodes T, Guess HA. All-cause mortality and vascular events among patients with rheumatoid arthritis, osteoarthritis, or no arthritis in the UK General Practice Research Database.J Rheumatol 2003;30:1196-202.

16 Haara MM, Heliövaara M, Kröger H, Arokoski JPA, Manninen P, Kärkkäinen A, et al. Osteoarthritis in the carpometacarpal joint of the thumb: prevalence and associations with disability and mortality. J Bone Joint Surg Am 2004;86:1452-7.

17 Robertsson 0, Stefansdottir A, Lidgren L, Ranstam J. Increased long term mortality in patients less than 55 years old who have undergone knee replacement for osteoarthritis: results from the Swedish Knee Arthroplasty Register. J Bone Joint Surg Br 2007;89:599-603.

18 Kumar N, Marshall NJ, Hammal DM, Pearce MS, Parker L, Furniss SS, et al. Causes of death in patients with rheumatoid arthritis: comparison with siblings and matched osteoarthritis controls. J Rheumatol 2007;34:1695-8.

19 Cisternas MG, Yelin E, Katz JN, Solomon DH, Wright EA, Losina E. Ambulatory visit utilization in a national, population-based sample of adults with osteoarthritis. Arthritis Rheum 2009;61:1694-703.

20 Frankel S, Eachus I, Pearson N, Greenwood R, Chan P, Peters TJ, et al. Population requirement for primary hip-replacement surgery: a cross-sectional study. Lancet 1999;353:1304-9.

21 Juni P, Dieppe P, Donovan J, Peters T, Eachus I, Pearson N, et al. Population requirement for primary knee replacement surgery: a cross-sectional study. Rheumatology 2003;42:516-21.
22 Eachus J, Williams M, Chan P, Smith GD, Grainge M, Donovan J, et al. Deprivation and cause specific morbidity: evidence from the Somerset and Avon survey of health. BMJ 1996;312:287-92

23 Juni P, Low N, Reichenbach S, Villiger PM, Williams S, Dieppe PA. Gender inequity in the provision of care for hip disease: populationbased cross-sectional study. Osteoarthritis Cartilage 2010;18:640-5.

24 Reichenbach S, Dieppe PA, Nuesch E, Williams S, Villiger PM, Juni P. Association of bone attrition with knee pain, stiffness and disability: a cross-sectional study. Ann Rheum Dis 2011;70:293-8.

25 Kellgren JH, Lawrence IS. Radiological assessment of osteo-arthrosis. Ann Rheum Dis 1957;16:494-502.

26 Royston P. Multiple imputation of missing values. Stata ) 2004; $4: 227-41$

27 Sterne JA, White IR, Carlin JB, Spratt M, Royston P, Kenward MG, et al Multiple imputation for missing data in epidemiological and clinical research: potential and pitfalls. BMJ 2009;338:b2393.

28 Van Buuren S, Boshuizen HC, Knook DL. Multiple imputation of missing blood pressure covariates in survival analysis. Stat Med 1999;18:681-94.

29 Felson DT, Chaisson CE, Hill CL, Totterman SM, Gale ME, Skinner KM, et al. The association of bone marrow lesions with pain in knee osteoarthritis. Ann Intern Med 2001;134:541-9.

30 Abramson SB, Attur M. Developments in the scientific understanding of osteoarthritis. Arthritis Res Ther 2009;11:227.

31 Stannus O, Jones G, Cicuttini F, Parameswaran V, Quinn S, Burgess J, et al. Circulating levels of IL-6 and TNF-alpha are associated with knee radiographic osteoarthritis and knee cartilage loss in older adults. Osteoarthritis Cartilage 2010;18:1441-7.

32 Couzin-Frankel J. Inflammation bares a dark side. Science 2010;330:1621.

33 Wagner P, Olsson H, Lidgren L, Robertsson O, Ranstam J. Increased cancer risks among arthroplasty patients: 30 year follow-up of the Swedish Knee Arthroplasty Register. Eur J Cancer 2011 Jan 10 [epub ahead of print].

34 Trelle S, Reichenbach S, Wandel S, Hildebrand P, Tschannen B, Villiger PM, et al. Cardiovascular safety of non-steroidal antiinflammatory drugs: network meta-analysis. BMJ 2011;342:c7086.

35 Lavie Cl, Milani RV, Ventura HO. Obesity and cardiovascular disease: risk factor, paradox, and impact of weight loss. I Am Coll Cardiol 2009;53:1925-32.

36 Choi HK, Curhan G. Independent impact of gout on mortality and risk for coronary heart disease. Circulation 2007;116:894-900.

37 Jordan KM, Cameron IS, Snaith M, Zhang W, Doherty M, Seckl J, et al. British Society for Rheumatology and British Health Professionals in Rheumatology guideline for the management of gout. Rheumatology 2007;46:1372-4.

38 Zhang W, Doherty M, Bardin T, Pascual E, Barskova V, Conaghan P, et al. EULAR evidence based recommendations for gout. Part II: management. Report of a task force of the EULAR Standing Committee for International Clinical Studies Including Therapeutics (ESCISIT). Ann Rheum Dis 2006;65:1312-24.

39 Wagenmakers R, van den Akker-Scheek I, Groothoff JW, Zijlstra W, Bulstra SK, Kootstra JW, et al. Reliability and validity of the short questionnaire to assess health-enhancing physical activity (SQUASH) in patients after total hip arthroplasty. BMC Musculoskelet Disord 2008;9:141.

40 Bellocco R, Jia C, Ye W, Lagerros YT. Effects of physical activity, body mass index, waist-to-hip ratio and waist circumference on total mortality risk in the Swedish National March Cohort. Eur J Epidemio 2010;25:777-88.

41 Holtermann A, Mortensen OS, Burr H, Sogaard K, Gyntelberg F, Suadicani P. Fitness, work, and leisure-time physical activity and ischaemic heart disease and all-cause mortality among men with pre-existing cardiovascular disease. Scand J Work Environ Health 2010;36:366-72.

42 Fransen M, McConnell S. Exercise for osteoarthritis of the knee. Cochrane Database Syst Rev 2008;4:CD004376.

43 Hernandez-Molina G, Reichenbach S, Zhang B, Lavalley M, Felson DT. Effect of therapeutic exercise for hip osteoarthritis pain: results of a meta-analysis. Arthritis Rheum 2008;59:1221-8.

44 Jenkinson CM, Doherty M, Avery AJ, Read A, Taylor MA, Sach TH, et al. Effects of dietary intervention and quadriceps strengthening exercises on pain and function in overweight people with knee pain: randomised controlled trial. BMJ 2009;339:b3170.

45 Penninx BW, Rejeski WJ, Pandya J, Miller ME, Di Bari M, Applegate WB, et al. Exercise and depressive symptoms: a comparison of aerobic and resistance exercise effects on emotional and physical function in older persons with high and low depressive symptomatology. J Gerontol B Psychol Sci Soc Sci 2002;57:P124-32.

Accepted: 17 January 2011 